\title{
WILEY-VCH
}

\section{Electron beam lithography of magnetic skyrmions}

Yao Guang, Yong Peng, Zhengren Yan, Yizhou Liu, Junwei Zhang, Xue Zeng, Senfu Zhang,

Shilei Zhang, David M. Burn, Nicolas Jaouen, Jinwu Wei, Hongjun Xu, Jiafeng Feng, Chi

Fang, Gerrit van der Laan, Thorsten Hesjedal, Baoshan Cui, Xixiang Zhang*, Guoqiang Yu*, Xiufeng Han

Y. Guang, Z. Yan, Dr. Y. Liu, Dr. J. Wei, Dr. H. Xu, Prof. J. Feng, C. Fang, Prof. G. Yu, Prof. X. Han

Beijing Natianal Laboratory for Condensed Matter Physics, Institute of Physics, Chinese Academy of Sciences, Beijing 100190, China

Email: guoqiangyu@iphy.ac.cn

Y. Guang, Z. Yan, Dr. Y. Liu, Dr. J. Wei, Dr. H. Xu, Prof. J. Feng, C. Fang, Prof. G. Yu, Prof. X. Han

Center of Materials Science and Optoelectronics Engineering, University of Chinese Academy of Sciences, Beijing 100049, China

Prof. Y. Peng, Dr. J. Zhang

Key Laboratory for Magnetism and Magnetic Materials of Ministry of Education, Lanzhou University, Lanzhou 730000, China

Dr. J. Zhang, Dr. S. Zhang, Prof. X. Zhang

Physical Science and Engineering Division (PSE), King Abdullah University of Science and Technology (KAUST), Thuwal 23955-6900, Saudi Arabia

Email: xixiang.zhang@kaust.edu.sa

Dr. X. Zeng

School of Mathematics and Physics, Lanzhou Jiaotong University, Lanzhou, 730070, China

Prof. S. Zhang

ShanghaiTech Laboratory for Topological Physics, ShanghaiTech University, Shanghai 201210, China

Prof. S. Zhang

School of Physical Science and Technology, ShanghaiTech University, Shanghai 201210, China

Dr. D. M. Burn, Prof. G. van der Laan

Magnetic Spectroscopy Group, Diamond Light Source, Didcot, OX11 0DE, UK

Dr. N. Jaouen

Synchrotron SOLEIL, L'Orme des Merisiers, Saint-Aubin, 91192 Gif-sur-Yvette, France

Prof. T. Hesjedal

Department of Physics, Clarendon Laboratory, University of Oxford, Parks Road, Oxford OX1 3PU, UK

Dr. J. Wei, Dr. H. Xu, Dr. B. Cui, Prof. G. Yu, Prof. X. Han

Songshan Lake Materials Laboratory, Dongguan, Guangdong 523808, China

Keywords: Magnetic skyrmion, electron beam lithography, transmission electron microscopy, skyrmion lattice 


\title{
WILEY-VCH
}

\begin{abstract}
The emergence of magnetic skyrmions, topological spin textures, has aroused tremendous interest in studying the rich physics related to their topology. While skyrmions promise highdensity and energy-efficient magnetic memory devices for information technology, the manifestation of their non-trivial topology through single skyrmions, ordered, and disordered skyrmion lattices could also give rise to many fascinating physical phenomena, such as the chiral magnon and skyrmion glass states. Therefore, generating skyrmions at designated locations on a large scale, while controlling the skyrmion patterns, is key to advancing topological magnetism. Here, we present a new, yet general, approach to the 'printing' of skyrmions with zero-field stability in arbitrary patterns on a massive scale in exchange-biased magnetic multilayers. By exploiting the fact that the antiferromagnetic order can be reconfigured by local thermal excitations, we use a focused electron beam with a graphic pattern generator to 'print' skyrmions, which we refer to as skyrmion lithography. Our work provides a route to design arbitrary skyrmion patterns, thereby establishing the foundation for further exploration of topological magnetism.
\end{abstract}




\section{WILEY-VCH}

The recently discovered topological magnetic skyrmion spin texture promises future innovative memory and logic devices owing to its nanoscale size and low driving current density [1-4]. Concerning their non-trivial real-space topology, skyrmions exhibit many intriguing properties [5-8], such as the topological Hall effect [6], the skyrmion Hall effect [9], and the rich ferromagnetic resonance states [10]. Furthermore, long-range ordered and disordered skyrmion lattices also give rise to fascinating phenomena like a chiral magnon state $[11,12]$ and skyrmion glass state [13], thus providing an arena for exploring topological physics[14, 15], nonreciprocal responses [16], and unconventional spintronic devices [17, 18]. To further elucidate the physics of skyrmions and their lattices, the crucial task is to create skyrmions in a controllable manner.

Magnetic skyrmions emerge as the ground state in specific types of magnetic materials that lack inversion symmetry in most cases with the presence of a magnetic field. For example, a long-range ordered skyrmion lattice typically appears in bulk magnetic materials with intrinsic inversion symmetry breaking (e.g., B20-type compounds), and its structure is usually restricted to a closely packed triangle lattice or square lattice $[1,2,19]$. On the other hand, in magnetic thin film heterostructures with interfacial inversion symmetry breaking [20-25], skyrmions are usually randomly distributed [25, 26], and long-range ordered skyrmion lattices are rarely observed. In both cases, skyrmions are formed at random positions after applying an external magnetic field in an uncontrolled manner.

In order to create skyrmions at a designated site, several methods have been successfully employed. First, an artificial skyrmion lattice can be obtained using modern nanofabrication technologies, such as pattering additional magnetic nano-disks on top of the skyrmion thin film [27-29], introducing structural defects in the films [30], and patterning confined structures [31], which can nucleate skyrmions at specified places and in particular configurations. Nevertheless, these methods permanently alter the local (or surrounding) magnetic properties of the sample, thereby smearing out their collective nature and limit their application merits. Recently, various 


\section{WILEY-VCH}

new methods for the local creation of skyrmions have been established, such as using a scanning tunneling microscopy tip [20, 32], a focused laser beam [33], as well as synchrotron X-rays [34]. However, the efficiency of skyrmion creation is very low, and all these methods are impractical in terms of device applications. To promote the exploitation of skyrmion lattices in fundamental research and practical application, it is thus crucial to realize artificial skyrmion lattices on a massive scale in a non-destructive and reconfigurable manner.

In this work, we demonstrate that a focused electron beam can be used to create individual skyrmions on demand in arbitrary positions in an exchange-biased magnetic multilayer (Fig. 1a). Starting by using Lorentz transmission electron microscopy (LTEM), where the skyrmion can be imaged in situ, we show that an electron beam can effectively create individual skyrmions. Then, we prove that by taking advantage of the graphical scanning function of the electron beam lithography (EBL) system, we can massively pattern zero-field-stable skyrmion lattices. This work will promote the exploration of collective phenomena in both ordered and disordered skyrmion lattices.

The studied samples consist of substrate/Pt $(5 \mathrm{~nm}) / \mathrm{Co}(0.6 \mathrm{~nm}) / \mathrm{IrMn}(5 \mathrm{~nm}) /[\mathrm{Pt}(2 \mathrm{~nm}) / \mathrm{Co}$ $(0.6 \mathrm{~nm}) / \mathrm{IrMn}(5 \mathrm{~nm})]_{11} / \mathrm{NiO}(2 \mathrm{~nm})($ Fig. $1 \mathrm{~b})$, which has an a perpendicular magnetic anisotropy (PMA) (see Fig. S1). Figure 1c shows the out-of-plane magnetic hysteresis $(M-H)$ loops for the as-grown and annealed samples. The as-grown sample exhibits a zero-centered $M-H$ loop, which has a wasp-waist shape. No exchange bias is observed in the as-grown sample, because there is no directional preference (no applied external field) during the deposition process. As a consequence, the magnetization direction of the PMA Co layer is randomly distributed up and down (see the labyrinthine domain structure in Fig. 1d and supplementary Fig. S2). Meanwhile, a randomly distributed local antiferromagnetic order (and thus a randomly distributed corresponding exchange bias), with $\sim 50 \%$ pointing up (positive field direction) and $\sim 50 \%$ pointing down (negative field direction), is also formed in the as-grown sample. Such a 


\section{WILEY-VCH}

random exchange bias distribution results in the wasp-waist-shaped hysteresis loop where the top (bottom) half is mostly shifted to the positive (negative) field direction due to the $\sim 50 \%$ negative (positive) exchange bias. After annealing at $200^{\circ} \mathrm{C}$ for 30 min under an out-of-plane magnetic field of $1 \mathrm{~T}$, the $M-H$ loop is shifted to the left (red dots), indicating the formation of an exchange bias $(69.2 \mathrm{mT})$ in the out-of-plane direction. The appearance of exchange bias is a result of the realignment of uncompensated antiferromagnetic spins at the $\mathrm{Co} / \mathrm{IrMn}$ interface from a random distribution to a uniform distribution during the field annealing process[35, 36].

We first show that, analogous to the thermal annealing process, the electron beam can also introduce a local exchange bias in the studied sample. The sample is illuminated in a TEM using bright-field mode at room temperature. During the illumination, an out-of-plane magnetic field of $209 \mathrm{mT}$ is applied to saturate the magnetization. After illuminating for $5 \mathrm{~min}$, the electron beam is turned off, and then the magnetic field is decreased to zero. Figure 1e shows the LTEM image of the illuminated area (labeled by the dashed circle). The illuminated area becomes a single domain, implying the formation of an exchange bias within this area. In sharp contrast to the illuminated area (Fig. 1d), the magnetic domain outside the illuminated area remains in the labyrinthine state. This result suggests that an exchange bias is introduced by electron beam illumination. The local $M-H$ loop of the illuminated area should be shifted towards negative direction, similar to that of the annealed sample (red dots in Fig. 1c). Hence, the single domain is stable at zero-field, corresponding to the case of point A in the $M-H$ loop in Fig. 1c.

Apart from a single domain, the electron beam illumination can also create zero-field single skyrmions. By adjusting the magnification of TEM to the maximum, the electron beam can be focused on a very small area ( $\sim 60 \mathrm{~nm}$ in diameter). After $3 \mathrm{~s}$ illumination on a selected position within the single domain background (Fig. 1e), the first skyrmion $(\sim 150 \mathrm{~nm}$ in diameter) is created, which is labeled '\#1' in Fig. 2a. The second skyrmion (\#2) and the other seven skyrmions are created in sequence in the same manner. One striking feature is that the created skyrmions are stable at zero field. This is different from the conventional skyrmion 


\section{WILEY-VCH}

materials, where the skyrmions need to be stabilized by an external magnetic field (see supplementary section S2) $[1,2,19-25,37]$. The zero-field skyrmion is the direct consequence of the out-of-plane exchange bias, which replace the role of the external magnetic field $[38,39]$. To create single skyrmions, the electron beam must be focused in a small area. Increasing the illumination area causes the formation of a large-sized multidomain pattern, as shown in Fig. 2b. The formed domain pattern resembles the as-grown magnetic state shown in Fig. 1d. Although the created skyrmions seem to possess an overall Néel-type domain wall, as indicated by comparing the images at different angles (see supplementary Fig. S4), our circular dichroic resonant elastic X-ray scattering results showed that the domain walls in the studied sample have a more complicated three-dimensional structure induced by dipole-dipole interaction $[40$, 41], as discussed in supplementary section S4.

A very focused electron beam can lead to significant thermal effects [42-46]. The creation of single skyrmions by the electron beam can thus be attributed to local heating. The local temperature increase causes the 'melting' of the exchange bias, resulting in the loss of singledomain stability of the local Co magnetic moments at zero-field. The effective field (the sum of the exchange, Dzyaloshinskii-Moriya interaction (DMI), perpendicular magnetic anisotropy, and dipole-dipole field) acting on the local Co layer causes the reversal of its magnetization, as shown in Fig. 2d. When the electron beam is turned off, the local antiferromagnetic order forms again according to the spin orientation of the adjacent Co layer. Because of the reversed Co magnetization in the illuminated area, the interfacial uncompensated spins are also reversed, as shown in Fig. 2e, resulting in a locally opposite exchange bias. We point out that the focused electron beam-induced heating does not damage the sample structure as manifested by the unchanged magnetic contract after repeating creation and elimination process.

We further carried out micromagnetic simulations to capture the heating-induced creation of skyrmion. The temperature at the illumination center is assumed to be maximum and to decrease radially to room temperature within $R \mathrm{~h}$ during electron beam illumination, as shown 


\section{WILEY-VCH}

in Fig. 2f. It should be noted that the area affected by the heating effect exceeds the illumination area due to the thermal diffusion (see supplementary section S5), which explains why the experimentally observed domain structure is larger than electron beam size. The exchange bias is assumed to change inversely with the temperature. The magnetic anisotropy, exchange constant, DMI, and saturation magnetization, which usually vary with the temperature[47], are assumed to be constant to better capture the role of local heating. The simulation results are shown in Figs. $2 \mathrm{~g}$ and $2 \mathrm{~h}$. When the modification of the exchange bias $(R \mathrm{~h}=50 \mathrm{~nm})$ is local, a single skyrmion can be created. As the influenced area becomes larger $(R \mathrm{~h}=400 \mathrm{~nm})$, a multidomain state is created, which is in agreement with the experimental results.

Although the local heating effect plays an important role in the skyrmion creation, we cannot completely exclude other possible origins, such as the influence of the electron excitation in the IrMn layer. During the creation of the single domain background (Fig. 1e), the focused area of electron beam is actually very large $(\sim 11 \mu \mathrm{m})$, notably decreasing the electron beam flux. As a result, the thermal effect is significantly reduced (see supplementary section S6). However, the reconstruction of the exchange bias can still be observed. We speculate that the electron beam could also lead to a non-thermal effect on IrMn, which plays a major role at low electron dose. The high-energy electrons interact with the atomic electrons surrounding the nucleus, giving rise to secondary electron production. The electron excitation in the antiferromagnet can in principle cause the reconstruction of antiferromagnetic order [48] or the change of anisotropy [49, 50].

In addition to creating individual skyrmions in the ferromagnetic layers, an interfacial local antiferromagnetic domain could also be created in the antiferromagnetic layers, as shown in Fig. 2e. To further prove the reversal of local antiferromagnetic order at the interface, the sample is imaged after recycling the external magnetic field, as illustrated in Figs. 3b-3d. Following the same procedure, a single domain background is first introduced and then four skyrmions are created (Fig. 3a) using the electron beam. After skyrmion creation, the magnetic 


\section{WILEY-VCH}

field is set to $200 \mathrm{mT}$ to saturate the magnetization. When the magnetic field decreases to zero, the background (except for the illuminated area) remains pointing up, corresponding to point $\mathrm{A}$ in Fig. 1c. In contrast, the four skyrmions with opposite core magnetization (pointing down) appear again at the same positions due to the locally reversed exchange bias (Fig. 3b). When the field is set to $-200 \mathrm{mT}$ and then back to zero (corresponding to point B in Fig. 1c), the film shows a labyrinthine domain pattern (Fig. 3c). The disappearance of the single domain background is because the exchange bias is not large enough to shift the entire $M-H$ loop to the negative side due to its finite coercivity. However, the same domain pattern shows up again when the magnetization is saturated at $200 \mathrm{mT}$ and returned to zero, as shown in Fig. 3d. An external magnetic field of up to $2 \mathrm{~T}$ (the maximum field available in our TEM) is also applied to the sample when the electron beam is off. The created skyrmions still appear after removing the external field, further demonstrating that the local antiferromagnetic domains are immune to the external magnetic field of up to $2 \mathrm{~T}$. The field sweeping results demonstrate the persistence of the locally modified exchange bias.

Although the above results demonstrate the creation of skyrmions in the studied samples, using a TEM is not practical for massive and large-area generation of skyrmions. We next prove that the skyrmions can also be created using the electron beam in an EBL system, which has been optimized for nano-sized device fabrication. Figures 4a-c depict the EBL-patterned square skyrmion lattices with different illumination duration obtained through magnetic force microscopy (MFM). Similar to the results obtained with TEM, the creation of skyrmion strongly depends on the duration of illumination. When the duration is short, the created circularly shaped domain in the illuminated area has a very weak but observable contrast. This can be explained by the reversal of several layers among the multilayer due to the insufficient illumination time for reversing the whole stacks. Such partial reversals can also be captured by micromagnetic simulations, as shown in supplementary section S6. With the increase of illumination time, the probability of a full skyrmion creation increases (see Fig. 4d). An 


\section{WILEY-VCH}

appropriate illumination duration can ensure the creation of a full stacking skyrmion. It is noted that the illumination duration for creating the different skyrmions is not identical, which is probably related to the nonuniformity of the exchange bias strength across the film due to the presence of antiferromagnetic domains[51].

The powerful capability of EBL lies in its precise scanning of the electron beam for generating arbitrary nanoscale patterns onto an electron-sensitive photoresist. The spatial resolution of scanning reaches down to sub-10 nm. Taking advantage of this capability, the lithography of magnetic skyrmions can be realized on the nanoscale. Figures 5a-c show the created long-range ordered artificial skyrmion lattices with lattice structures of square, kagome, and honeycomb arrangements. The realization of various structures lays the foundation for exploring the chiral magnon state in the skyrmion lattice. Figure $5 \mathrm{~d}$ also shows a disordered skyrmion lattice created by EBL. Although we only show four representative skyrmion patterns, any arrangement of skyrmion can be realized. Compared to TEM, the electron acceleration voltage of EBL is significantly reduced. Even so, the creation of skyrmion using EBL is still very effective. We speculate that the film on a thick $\mathrm{Si} / \mathrm{SiO}_{2}$ substrate may reduce the transmission and enhance the backward electron scattering, resulting in equally efficient transfer from electron energy to thermal energy.

Using EBL for pattering skyrmion lattices presents a huge step forward towards enabling research on arbitrary skyrmion arrangements. To date, EBL has been well employed in pattering nanosized real structures combined with etching and lift-off processes. Our study allows for the direct lithography of magnetic structures in continuous films without the need for any additional, materials-altering steps. Compared to other optical manipulation of magnetization, the advantage of using an electron beam lies in its ultra-short wavelength and its turnability through emission voltage, which may allow for the manipulation of sub-10 nm magnetic domain. In this study, the size of the skyrmion, created using an electron beam, is $~ 150 \mathrm{~nm}$. We note that the size of these skyrmions is mainly limited by the intrinsic materials parameters rather than by 


\section{WILEY-VCH}

the size of the electron beam. The obtained skyrmion size is comparable to those of zero-field skyrmions in the previously studied extended multilayers that have a periodic structure $[26,52,53]$. Recently, zero-field magnetic skyrmions with a mean diameter of $\sim 60 \mathrm{~nm}$ have been realized at room temperature[39], indicating the potential to further reducing the skyrmion size in the studied materials through engineering the material parameters.

Our technique can be easily applied to other ferromagnet/antiferromagnet multilayers as well. The ease of lattice construction provides us the flexibility of studying the exploration of lattice-relevant physics [54], such as the emergence of chiral edge state and skyrmion glass state $[11,12,55]$. In addition to creating skyrmions in the perpendicularly magnetized thin films, this technique may also have an impact on the study of magnonics. In fact, this technique could be used to engineer magnetic domain patterns for exploiting the active manipulation of spin waves[56-58], analogous to the pioneering work on patterning specific in-plane magnetic domain structures via advanced scanning probe-based technique[56, 59]. Moreover, the reported technique provides the possibility of manipulating local antiferromagnetic domain, which will promote the study on antiferromagnetic skyrmion and perhaps the development of related devices. It is noted that all the experiments performed in the EBL system are under zero magnetic field, since the existing EBL system does not allow for the application of a magnetic field. To expand the capabilities of the developed technique presented in this work, and for enabling corresponding applications, developing an EBL system capable of providing magnetic fields is of great importance. 


\section{WILEY-VCH}

\section{Materials and Methods}

\section{Experimental Details}

The sample, consisting of $\operatorname{Pt}(5 \mathrm{~nm}) / \mathrm{Co}(0.6 \mathrm{~nm}) / \operatorname{Ir} 22 \mathrm{Mn} 78(\operatorname{IrMn})(5 \mathrm{~nm}) /[\operatorname{Pt}(2 \mathrm{~nm}) / \operatorname{Co}(0.6$ $\mathrm{nm}) / \mathrm{IrMn}(5 \mathrm{~nm})]_{11} / \mathrm{NiO}(2 \mathrm{~nm})$, was grown on silicon nitride membranes and a $\mathrm{Si} / \mathrm{SiO}_{2}$ substrate using a magnetron sputtering system. The $\mathrm{Si} / \mathrm{Si} \mathrm{N}_{4}$ membranes and $\mathrm{Si} / \mathrm{SiO}_{2}$ wafers were used as substrates for carrying out Lorentz transmission electron microscopy (LTEM) and magnetic force microscopy (MFM) measurements, respectively. The background vacuum in the sputtering system was $4.75 \times 10_{-6} \mathrm{~Pa}$. The deposition rates for Pt, Co, and IrMn were $4.18 \AA$ \&/s, $1.91 \AA / \mathrm{s}$, and $7.77 \AA / \mathrm{s}$, respectively, at a power of $100 \mathrm{~W}$ and pressure of $0.16 \mathrm{~Pa}$. The deposition rate for $\mathrm{NiO}$ was $0.92 \AA / \mathrm{s}$ at a power of $100 \mathrm{~W}$ and pressure of $1.5 \mathrm{~Pa}$. For the annealing sample, an out-of-plane field of $1 \mathrm{~T}$ was applied at $200^{\circ} \mathrm{C}$ under $5 \times 10_{-4} \mathrm{~Pa}$ for 30 min. The LTEM images were acquired in a FEI Titan Cs Probe TEM in Lorentz mode (the Fresnel imaging mode) at $300 \mathrm{kV}$. All images were obtained at a tilted angle and defocus of $600 \mu \mathrm{m}$. The out-of-plane magnetic field can be applied and tuned by varying the current passing through the microscope's objective lens. A commercial electron beam lithography system (Eline plus, Raith, Germany) was used under an exposure mode to pattern the skyrmion arrays. The acceleration voltage was $10 \mathrm{kV}$, and the aperture is $30 \mu \mathrm{m}$. The skyrmion was created via the dot exposure mode. The beam current was fixed at $0.231 \mathrm{nA}$ and the dot dose is changed by varying the exposure time. The major difference in creating skyrmions between TEM and EBL is the scanning method. The scanning was performed in manual mode in TEM, but in programmable manner in the EBL instrument. All MFM images were acquired in a Dimension scanning probe microscopy system (D3000, Veeco, USA) at room temperature in zero external field. The magnetic response was studied using standard magnetic $\mathrm{Co} / \mathrm{Al}$-coated cantilevers (PPP-MFMR, Nanosensors, $2.8 \mathrm{~N} / \mathrm{m}$ spring constant) in phase mode and with a lift height of $30 \mathrm{~nm}$. 


\section{WILEY-VCH}

\section{Micromagnetic Simulations}

The micromagnetic simulations were carried out using Mumax3 [60]. A magnetic multilayer with 10 repetitions was simulated. The geometry of each magnetic layer is $2000 \mathrm{~nm} \times 2000 \mathrm{~nm}$ $\times 0.6 \mathrm{~nm}$ with a mesh size of $8 \mathrm{~nm} \times 8 \mathrm{~nm} \times 0.6 \mathrm{~nm}$ and open boundary conditions. The different magnetic layers are separated by a $6.6 \mathrm{~nm}$ spacer. Exchange interaction, Dzyaloshinskii-Moriya interaction (DMI), perpendicular magnetic anisotropy (PMA), Zeeman interaction, and magnetic dipole-dipole interaction are taken into account in the simulations. The following parameters were used in the simulations: exchange constant $A=10 \mathrm{pJ} / \mathrm{m}, \mathrm{DMI}$ constant $D=$ $1.35 \mathrm{~mJ} / \mathrm{m} 2$, PMA constant $K \mathrm{u}=965 \mathrm{~kJ} / \mathrm{m}_{3}$, saturation magnetization $M \mathrm{~s}=1150 \mathrm{kA} / \mathrm{m}$ and Gilbert damping constant $\alpha=0.2$. An out-of-plane external field, $B$ ext $=68.7 \mathrm{mT}$, is applied to simulate the exchange bias effect of IrMn.

In our work, we assume that only the exchange bias in the illumination area changes due to the temperature increase. Because the temperature profile in the illumination area is nearly linear (see supplementary Fig. S6), we use a linear temperature profile for the investigation of the skyrmion nucleation by micromagnetic simulation. A circular region with a $50 \mathrm{~nm}(400 \mathrm{~nm})$ radius is used as the small (large) electron beam illumination region. The temperature outside the illumination region is assumed to be $300 \mathrm{~K}$ while a linear temperature gradient is employed inside the circle with a center temperature of $400 \mathrm{~K}(390 \mathrm{~K})$ for the small (large) circle to mimic the corresponding heating effect.

\section{Acknowledgments}

Y.G., Y.P, and Z.R.Y. have contributed equally to this work. Financial support from the National Key Research and Development Program of China (Grants No. 2017YFA0206200), the National Natural Science Foundation of China (NSFC, Grants No. 11874409, 11804380, 51801087), Beijing Natural Science Foundation (Grant No. Z190009), the Strategic Priority Research Program (B) [Grant No. XDB07030200], the Key Research Program of Frontier 


\section{WILEY-VCH}

Sciences (Grant No. QYZDJ-SSW-SLH016), the International Partnership Program (Grant No.

112111KYSB20170090) of the Chinese Academy of Sciences (CAS), K. C. Wong Education Foundation (GJTD-2019-14), and Fujian Innovation Academy, Chinese Academy of Sciences (Grant No. FJCXY18040302). J.Z. and X.Z. acknowledge the financial support from the King Abdullah University of Science and Technology (KAUST), Office of Sponsored Research (OSR) under the Award No. OSR-2017-CRG6-3427. S.L.Z. acknowledges the starting grant from ShanghaiTech University and the Eastern Scholar Scheme. T.H. gratefully acknowledges support from EPSRC (EP/N032128/1). We acknowledge beamtime on beamline I10 at Diamond Light Source (Didcot, UK) under proposals SI20183 and MM21868, and on the SEXTANTS beamline at the SOLEIL synchrotron (Gif-sur-Yvette, France) under proposal 20181882. Guoqiang Yu acknowledges helpful discussions with Haifeng Du and Junjie Li.

\section{Author contributions}

G.Q.Y. conceived the project. Y.G. prepared the samples, characterized the multilayer films, performed the EBL patterning experiments, and carried out the MFM measurements with supports from G.Y., J.W., H.X., J.F., and C.F.. J.Z. and X.Z. performed the Lorentz TEM imaging experiments with supports from G.Y., Y.G., S.Z., Y.P., and X.Z.. S.L.Z., D.B., N.J., G.L., and T.H. carried out the CD-REXS experiments. Z.Y. and Y.L. performed the micromagnetic simulations with support from G.Y.. G.Y. drafted the paper and all authors commented on the manuscript. The study was performed under the supervision of G.Y. and X.H.. Y.G., Y. P.., and Z.Y. contributed equally to this research.

\section{Competing interests:}

The authors declare no competing interests.

\section{Data and materials availability:}


WILEY-VCH

All data needed to evaluate the conclusions in the paper are present in the paper. Additional data available from authors upon request. 


\section{WILEY-VCH}

[1] S. Mühlbauer, B. Binz, F. Jonietz, C. Pfleiderer, A. Rosch, A. Neubauer, R. Georgii, P. Boni, Science 2009, 323, 915.

[2] X. Z. Yu, Y. Onose, N. Kanazawa, J. H. Park, J. H. Han, Y. Matsui, N. Nagaosa, Y. Tokura, Nature 2010, 465, 901.

[3] F. Jonietz, S. Muhlbauer, C. Pfleiderer, A. Neubauer, W. Munzer, A. Bauer, T. Adams, R. Georgii, P. Boni, R. A. Duine, K. Everschor, M. Garst, A. Rosch, Science 2010, 330, 1648.

[4] X. Z. Yu, N. Kanazawa, W. Z. Zhang, T. Nagai, T. Hara, K. Kimoto, Y. Matsui, Y. Onose, Y. Tokura, Nat. Commun. 2012, 3, 988.

[5] A. Fert, N. Reyren, V. Cros, Nat. Rev. Mater. 2017, 2, 17031.

[6] N. Nagaosa, Y. Tokura, Nat. Nanotechnol. 2013, 8, 899.

[7] W. J. Jiang, G. Chen, K. Liu, J. D. Zang, S. G. E. te Velthuis, A. Hoffmann, Phys. Rep. 2017, 704, 1.

[8] K. Everschor-Sitte, J. Masell, R. M. Reeve, M. Klaui, J. Appl. Phys. 2018, 124, 240901.

[9] J. D. Zang, M. Mostovoy, J. H. Han, N. Nagaosa, Phys. Rev. Lett. 2011, 107, 136804.

[10] Y. Onose, Y. Okamura, S. Seki, S. Ishiwata, Y. Tokura, Phys. Rev. Lett. 2012, 109, 037603.

[11] S. K. Kim, Y. Tserkovnyak, Phys. Rev. Lett. 2017, 119, 077204.

[12] Z. X. Li, C. Wang, Y. S. Cao, P. Yan, Phys. Rev. B 2019, 98, 180407(R).

[13] S. Hoshino, N. Nagaosa, Phys. Rev. B 2018, 97, 024413.

[14] K. Hamamoto, M. Ezawa, N. Nagaosa, Phys. Rev. B 2015, 92, 115417.

[15] K. Manna, Y. Sun, L. Muechler, J. Kubler, C. Felser, Nat. Rev. Mater. 2018, 3, 244.

[16] Y. Tokura, N. Nagaosa, Nat. Commun. 2018, 9, 3740.

[17] Y. Q. Huang, W. Kang, X. C. Zhang, Y. Zhou, W. S. Zhao, Nanotechnology 2017, 28, 08LT02.

[18] K. M. Song, J. S. Jeong, B. Pan, X. C. Zhang, J. Xia, S. Cha, T. E. Park, K. Kim, S. Finizio, J. Raabe, J. Chang, Y. Zhou, W. S. Zhao, W. Kang, H. S. Ju, S. Woo, Nat. Electron. 2020, 3, 148 .

[19] K. Karube, J. S. White, N. Reynolds, J. L. Gavilano, H. Oike, A. Kikkawa, F. Kagawa, Y. Tokunaga, H. M. Rønnow, Y. Tokura, Y. Taguchi, Nat. Mater. 2016, 15, 1237.

[20] N. Romming, C. Hanneken, M. Menzel, J. E. Bickel, B. Wolter, K. von Bergmann, A. Kubetzka, R. Wiesendanger, Science 2013, 341, 636.

[21] S. Heinze, K. von Bergmann, M. Menzel, J. Brede, A. Kubetzka, R. Wiesendanger, G. Bihlmayer, S. Blugel, Nat. Phys. 2011, 7, 713.

[22] W. Jiang, P. Upadhyaya, W. Zhang, G. Yu, M. Jungfleisch, F. Fradin, J. Pearson, Y. Tserkovnyak, K. Wang, O. Heinonen, S. te Velthuis, A. Hoffmann, Science 2015, 349, 283.

[23] C. Moreau-Luchaire, C. Moutafis, N. Reyren, J. Sampaio, C. Vaz, N. Van Horne, K. Bouzehouane, K. Garcia, C. Deranlot, P. Warnicke, P. Wohlhuter, J. George, M. Weigand, J. Raabe, V. Cros, A. Fert, Nat. Nanotechnol. 2016, 11, 731.

[24] S. Woo, K. Litzius, B. Kruger, M. Im, L. Caretta, K. Richter, M. Mann, A. Krone, R. Reeve, M. Weigand, P. Agrawal, I. Lemesh, M. Mawass, P. Fischer, M. Klaui, G. Beach, Nat. Mater. 2016, 15, 501.

[25] G. Yu, P. Upadhyaya, X. Li, W. Li, S. K. Kim, Y. Fan, K. L. Wong, Y. Tserkovnyak, P. K. Amiri, K. L. Wang, Nano Lett. 2016, 16, 1981.

[26] S. F. Zhang, J. W. Zhang, Q. Zhang, C. Barton, V. Neu, Y. L. Zhao, Z. P. Hou, Y. Wen, C. Gong, O. Kazakova, W. H. Wang, Y. Peng, D. A. Garanin, E. M. Chudnovsky, X. X. Zhang, Appl. Phys. Lett. 2018, 112, 132405.

[27] L. Sun, R. X. Cao, B. F. Miao, Z. Feng, B. You, D. Wu, W. Zhang, A. Hu, H. F. Ding, Phys. Rev. Lett. 2013, 110, 167201

[28] J. Li, A. Tan, K. W. Moon, A. Doran, M. A. Marcus, A. T. Young, E. Arenholz, S. Ma, R. F. Yang, C. Hwang, Z. Q. Qiu, Nat. Commun. 2014, 5, 4704. 


\section{WILEY-VCH}

[29] D. A. Gilbert, B. B. Maranville, A. L. Balk, B. J. Kirby, P. Fischer, D. T. Pierce, J. Unguris, J. A. Borchers, K. Liu, Nat. Commun. 2015, 6, 8462.

[30] S. Zhang, A. K. Petford-Long, C. Phatak, Sci. Rep. 2016, 6, 31248.

[31] J. Brandao, D. A. Dugato, M. V. P. dos Santos, J. C. Cezar, ACS Appl. Nano Mater. 2019, 2, 7532.

[32] P. J. Hsu, A. Kubetzka, A. Finco, N. Romming, K. von Bergmann, R. Wiesendanger, Nat. Nanotechnol. 2017, 12, 123.

[33] S. G. Je, P. Vallobra, T. Srivastava, J. C. Rojas-Sanchez, T. H. Pham, M. Hehn, G. Malinowski, C. Baraduc, S. Auffret, G. Gaudin, S. Mangin, H. Bea, O. Boulle, Nano Lett. 2018, $18,7362$.

[34] Y. Guang, I. Bykova, Y. Liu, G. Yu, E. Goering, M. Weigand, J. Gräfe, S. K. Kim, J. Zhang, H. Zhang, Z. Yan, C. Wan, J. Feng, X. Wang, C. Guo, H. Wei, Y. Peng, Y. Tserkovnyak, X. Han, G. Schütz, Nat. Commun. 2020, 11, 949.

[35] J. Sort, V. Baltz, F. Garcia, B. Rodmacq, B. Dieny, Phys. Rev. B 2005, 71, 054411.

[36] Y. Qin, Y. F. Liu, AIP Adv. 2018, 8, 025314.

[37] A. O. Leonov, T. L. Monchesky, N. Romming, A. Kubetzka, A. N. Bogdanov, R. Wiesendanger, New J Phys 2016, 18, 065003.

[38] G. Q. Yu, A. Jenkins, X. Ma, S. A. Razavi, C. L. He, G. Yin, Q. M. Shao, Q. L. He, H. Wu, W. J. Li, W. J. Jiang, X. F. Han, X. Q. Li, A. C. B. Jayich, P. K. Amiri, K. L. Wang, Nano Lett. 2018, 18, 980.

[39] K. G. Rana, A. Finco, F. Fabre, S. Chouaieb, A. Haykal, L. D. Buda-Prejbeanu, O. Fruchart, S. Le Denmat, P. David, M. Belmeguenai, T. Denneulin, R. E. Dunin-Borkowski, G. Gaudin, V. Jacques, O. Boulle, Phys Rev Appl 2020, 13, 044079.

[40] W. Legrand, J. Y. Chauleau, D. Maccariello, N. Reyren, S. Collin, K. Bouzehouane, N. Jaouen, V. Cros, A. Fert, Sci. Adv. 2018, 4, eaat0415.

[41] W. Li, I. Bykova, S. Zhang, G. Yu, R. Tomasello, M. Carpentieri, Y. Liu, Y. Guang, J. Grafe, M. Weigand, D. M. Burn, G. van der Laan, T. Hesjedal, Z. Yan, J. Feng, C. Wan, J. Wei, X. Wang, X. Zhang, H. Xu, C. Guo, H. Wei, G. Finocchio, X. Han, G. Schutz, Adv. Mater. 2019, 31, e1807683.

[42] T. Yokota, M. Murayama, J. M. Howe, Phys. Rev. Lett. 2003, 91, 265504.

[43] R. F. Egerton, P. Li, M. Malac, Micron 2004, 35, 399.

[44] A. Reguer, F. Bedu, S. Nitsche, D. Chaudanson, B. Detailleur, H. Dallaporta, Ultramicroscopy 2009, 110, 61.

[45] H. M. Zheng, J. B. Rivest, T. A. Miller, B. Sadtler, A. Lindenberg, M. F. Toney, L. W. Wang, C. Kisielowski, A. P. Alivisatos, Science 2011, 333, 206.

[46] J. J. Li, Y. P. Li, Q. Li, Z. C. Wang, F. L. Deepak, Nanoscale Horiz. 2019, 4, 1302.

[47] R. Tomasello, K. Y. Guslienko, M. Ricci, A. Giordano, J. Barker, M. Carpentieri, O. Chubykalo-Fesenko, G. Finocchio, Phys. Rev. B 2018, 97.

[48] H. Ehrke, R. I. Tobey, S. Wall, S. A. Cavill, M. Forst, V. Khanna, T. Garl, N. Stojanovic, D. Prabhakaran, A. T. Boothroyd, M. Gensch, A. Mirone, P. Reutler, A. Revcolevschi, S. S. Dhesi, A. Cavalleri, Phys. Rev. Lett. 2011, 106, 217401.

[49] N. P. Duong, T. Satoh, M. Fiebig, Phys. Rev. Lett. 2004, 93, 117402.

[50] X. Ma, F. Fang, Q. Li, J. Zhu, Y. Yang, Y. Z. Wu, H. B. Zhao, G. Lupke, Nat. Commun. 2015, 6, 8800 .

[51] V. Baltz, J. Sort, S. Landis, B. Rodmacq, B. Dieny, Phys. Rev. Lett. 2005, 94.

[52] M. He, L. C. Peng, Z. Z. Zhu, G. Li, J. W. Cai, J. Q. Li, H. X. Wei, L. Gu, S. G. Wang,

T. Y. Zhao, B. G. Shen, Y. Zhang, Appl. Phys. Lett. 2017, 111, 202403.

[53] N. K. Duong, M. Raju, A. P. Petrovic, R. Tomasello, G. Finocchio, C. Panagopoulos, Appl. Phys. Lett. 2019, 114, 072401.

[54] F. Ma, Y. Zhou, H. B. Braun, W. S. Lew, Nano Lett. 2015, 15, 4029.

[55] S. A. Diaz, J. Klinovaja, D. Loss, Phys. Rev. Lett. 2019, 122, 187203. 


\section{WILEY-VCH}

[56] E. Albisetti, D. Petti, M. Pancaldi, M. Madami, S. Tacchi, J. Curtis, W. P. King, A. Papp, G. Csaba, W. Porod, P. Vavassori, E. Riedo, R. Bertacco, Nat. Nanotechnol. 2016, 11, 545.

[57] E. Albisetti, D. Pettio, G. Sala, R. Silvani, S. Tacchi, S. Finizio, S. Wintz, A. Calo, X. R. Zheng, J. Raabe, E. Riedo, R. Bertacco, Communications Physics 2018, 1.

[58] E. Albisetti, S. Tacchi, R. Silvani, G. Scaramuzzi, S. Finizio, S. Wintz, C. Rinaldi, M. Cantoni, J. Raabe, G. Carlotti, R. Bertacco, E. Riedo, D. Petti, Adv. Mater. 2020, 32.

[59] E. Albisetti, A. Calo, M. Spieser, A. W. Knoll, E. Riedo, D. Petti, Appl. Phys. Lett. 2018, 113.

[60] A. Vansteenkiste, J. Leliaert, M. Dvornik, M. Helsen, F. Garcia-Sanchez, B. Van Waeyenberge, AIP Adv. 2014, 4, 107133. 


\section{WILEY-VCH}

a

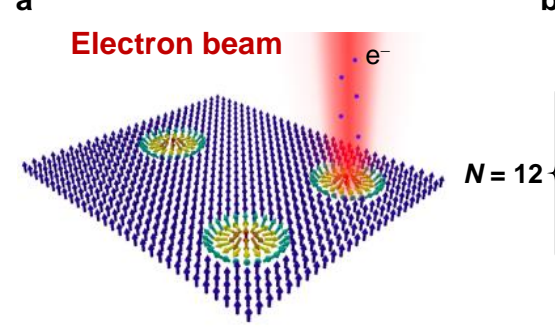

d

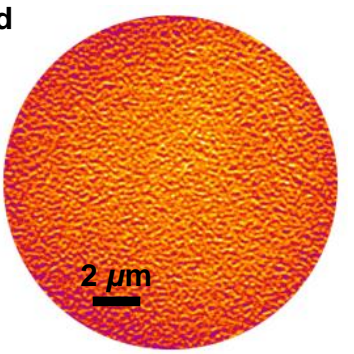

b

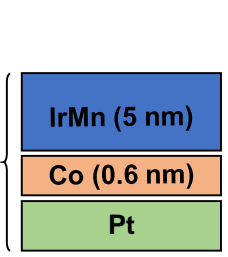

e

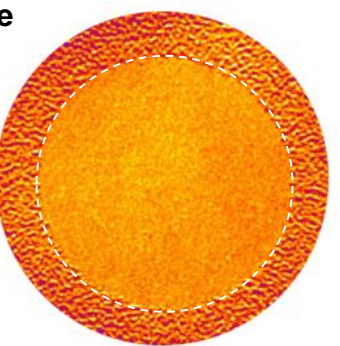

c

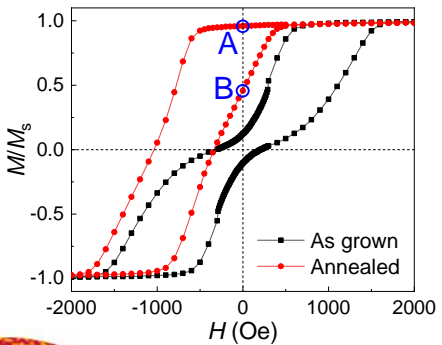

Figure 1. Sample properties and influence of electron beam illumination. a, Sketch of the creation of skyrmions using an electron beam. b, Core sample structure: $\mathrm{Pt}(5 \mathrm{~nm}) / \mathrm{Co}(0.6 \mathrm{~nm}) /$ $\operatorname{Ir} 22 \operatorname{Mn} 78(5 \mathrm{~nm}) /[\mathrm{Pt}(2 \mathrm{~nm}) / \mathrm{Co}(0.6 \mathrm{~nm}) / \operatorname{IrMn}(5 \mathrm{~nm})]_{11} / \mathrm{NiO}(2 \mathrm{~nm}) . \mathbf{c}$, Magnetic hysteresis loops for as-grown and annealed samples. Points A and B label the remanent magnetization states of the annealed sample for the magnetic field reduced from positive and negative saturation, respectively. d, LTEM image of a magnetic domain at zero field for the as-grown sample. The sample is tilted by $28.2^{\circ}$. The domain pattern imaged by LTEM is consistent with that obtained by MFM (see supplementary Fig. S2). e, LTEM image of the magnetic domain pattern at zero field after the circled area is illuminated for 5 min under a saturating magnetic field. The exposed central area becomes a single domain after illumination. The electron beam illumination is performed in the TEM system, where the working voltage is $200 \mathrm{keV}$. The dose rate for forming the circle single domain is $2.7 \mathrm{e}-/ \mathrm{nm}_{2} \mathrm{~s}$. The dose rate for imaging the magnetic domain is $2.4 \mathrm{e}-/ \mathrm{nm} 2 \mathrm{~s}$. 


\section{WILEY-VCH}

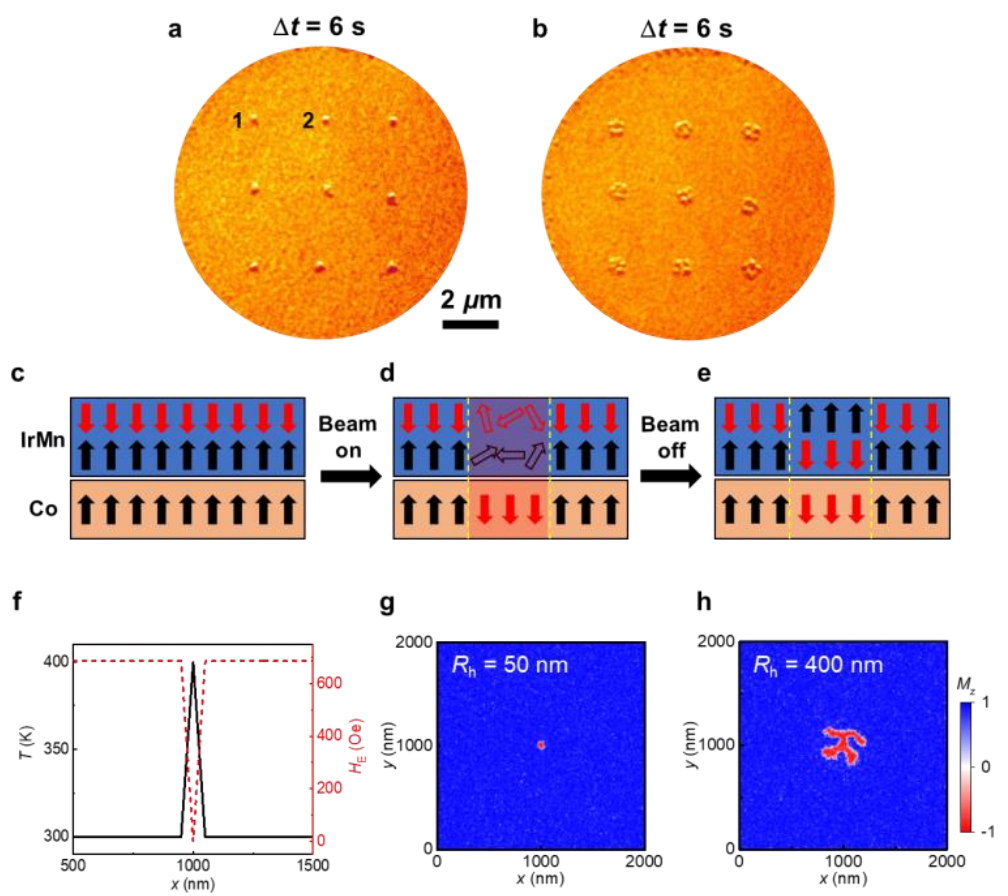

Figure 2. Skyrmion creation through local electron beam illumination. a, Electron-beaminduced magnetic domain pattern with an illumination time of $6 \mathrm{~s}$. The illuminated area is the smallest we can achieve ( $60 \mathrm{~nm}$ in diameter), corresponding to maximum magnification. $\mathbf{b}$, The illuminated area is about two times the size compared with a. c-e, Illustration of the skyrmion creation process. The magnetization of $\mathrm{Co}$ is in a single domain state under the exchange bias (c). During the electron beam illumination, the local exchange bias is broken due to the temperature rise (d). The local magnetization of Co is flipped during the loss of exchange bias. The local exchange bias is restored, however, with reversed orientation, after the electron beam is switched off (e). The black and red arrows represent the magnetization pointing up and down, respectively. The dashed yellow lines indicate the local region affected by the electron beam. f, Temperature profile around the illuminated region. $\mathbf{g , h}$, Micromagnetic simulation results for illumination area with a diameter of $100 \mathrm{~nm}$ and $800 \mathrm{~nm}$, respectively. 


\section{WILEY-VCH}

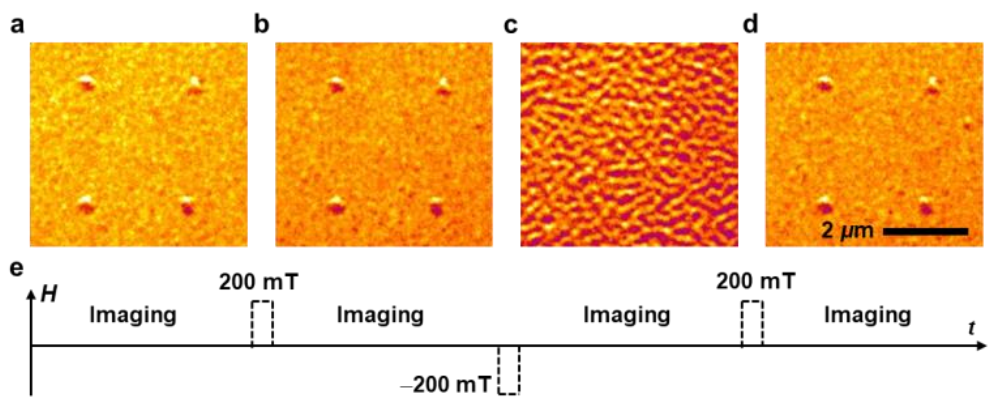

Figure 3. Demonstration of the created antiferromagnetic domain. a, LTEM images of skyrmions at zero field. Four skyrmions are sequentially created in the single domain background using a focused electron beam. b-d, LTEM images of domain patterns captured at zero magnetic field after firstly setting the magnetic field to $200 \mathrm{mT}(\mathbf{b}),-200 \mathrm{mT}$ (c), and 200 $\mathrm{mT}(\mathbf{d}) . \mathbf{e}$, The time sequence of applying the magnetic field and imaging process.
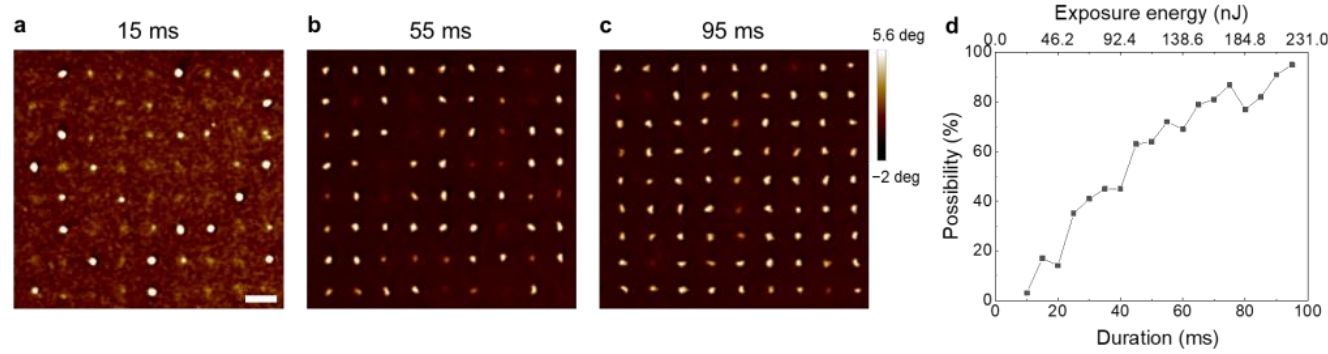

Figure 4. Artificial skyrmion lattice patterned by electron-beam lithography. a-c, MFM images of the artificial skyrmion lattices with illumination time of 15, 55, and $95 \mathrm{~ms}$. The scale bar is $1 \mu \mathrm{m}$. d, Probability of skyrmion creation as a function of illumination time. The working voltage of EBL is $10 \mathrm{keV}$.
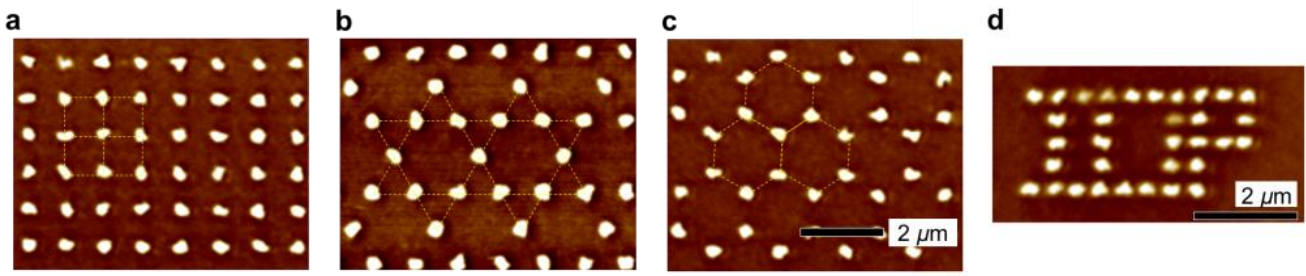


\section{WILEY-VCH}

Figure 5. a-c, MFM images of EBL-patterned artificial skyrmion lattices with square, kagome, and honeycomb configuration. The dashed yellow lines illustrate the structure of skyrmion lattice. d, MFM images of EBL-patterned disordered skyrmion lattice. The illumination time for the generation of an individual skyrmion is $250 \mathrm{~ms}$. 


\section{WILEY-VCH}

Table of Content: A new, yet general, approach to the 'printing' of skyrmions with zero-field stability in arbitrary patterns is demonstrated. By exploiting the fact that the antiferromagnetic order can be reconfigured by local thermal excitations, a focused electron beam with a graphic pattern generator is used to 'print' skyrmions, which is referred to as skyrmion lithography. Our work provides a route to design arbitrary skyrmion patterns, thereby establishing the foundation for further exploration of topological magnetism.

ToC figure

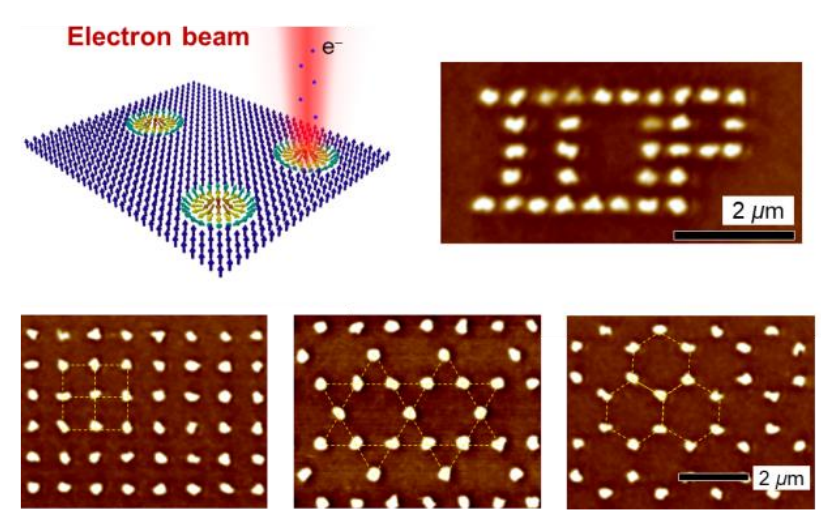

